\title{
Possibilité de suivi par télédétection spatiale de l'évolution de la végétation en zone de carrières
}

\author{
Sylvain PASQUIER \& Colette M. GIRARD \\ I.N.A. Paris-Grignon, Laboratoire de Géobotanique, F 78850 Thiverval-Grignon
}

RÉSUMÉ

\begin{abstract}
Les missions aériennes régulières sont beaucoup trop coûteuses pour permettre un repérage et un suivi satisfaisant des zones d'exploitation de carrières. La possibilité d'utiliser les données fournies par la télédétection spatiale a été testée à partir de 2 images : l'une de LANDSAT TM d'avril 1984, l'autre de SPOT d'août 1986. La réalisation de compositions colorées multidates et d'images classées diachroniques, basées sur une différence de 2 classifications assistées, effectuées à partir d'un même référentiel de sites-tests, semble constituer une méthode opérationnelle de suivi de l'évolution de l'occupation du sol. L'extension des zones dénudées, des plans d'eau, des surfaces défrichées et des terrains revégétalisés a pu être mise en évidence, cartographiée à l'échelle du $1 / 50000$, et quantifiée. La méthode mise en place pourrait être appliquée à d'autres sites de carrières, mais aussi, plus généralement au suivi de la gestion de zones en friches en milieu péri-urbain.
\end{abstract}

Mots clés additionnels : Friche, remblais, environnement, image satellite, compositions colorées multidates, image diachronique.

Possible remote sensing monitoring of vegetation evolution in quarry sites.

Regular aerial missions are too much expensive to allow satisfactory detection of quarry sites or monitoring of their use, and we have tested the possible use of satellite data for 2 images for this purpose : LANDSAT TM in April 1984, SPOT in August 1986. Multidate color composites, and diachronic classified images, based on the difference between 2 assisted classifications produced from the same test site references, seemed to form an operational method for surveying the use of quarries. Bare soil areas, water stands, cleared areas, and vegetated land were recognized and mapped at a 1/50000 scale, and evaluated. The method proposed could be applied to quarry areas generally, and also more widely to surveys of waste land around cities.

Additional key words : Waste, embankment, environment, satellite imagery, multidate color composites, diachronic image.

\section{INTRODUCTION}

Dans notre société, de plus en plus sensibilisée à la qualité de son environnement, l'exploitation des carrières est fréquemment ressentie comme une source de nuisances et une atteinte irréversible au paysage. Pour tenter de limiter l'impact sur le milieu naturel de cette activité, indispensable au bon fonctionnement de

Ce travail a été réalisé dans le cadre de l'attribution du diplôme d'Ingénieur Agronome, de l'INA PG, à l'Institut d'Aménagement et d'Urbanisme de la Région Ile-de-France, Division Environnement Urbain et Rural, rue de Vaugirard, F 75015 Paris. l'économie des pays industrialisés, des textes législatifs ont été promulgués dans les années 1970 afin de rendre obligatoire la remise en état des anciennes carrières par l'exploitant lui-même (COLMENERo, 1980). Par ailleurs des études récentes ont montré que l'on dispose aujourd'hui des connaissances et des techniques nécessaires pour effectuer des réaménagements de qualité (FEDOROFF, 1977 ; SAUVETERRE, 1985).

Pourtant le constat que l'on peut dresser sur le terrain est loin d'être positif. Deux phénomènes expliquent cette apparente contradiction :

- l'absence de schéma d'aménagement prévoyant pour l'ensemble des carrières d'une zone donnée, les réaménagements à effectuer en fonction des autres occupations du sol existantes ; 
- l'absence d'un suivi suffisamment rigoureux de la végétation implantée et spontanée, poussant sur les sols remis en place en fin d'exploitation. En effet cette végétation juvénile est particulièrement sensible aux ravageurs, aux maladies, aux accidents climatiques, et l'évolution de sa flore est souvent imprévisible (BOURNERIAS, 1959).

Face à ces carences nous avons étudié les possibilités d'utiliser la télédétection spatiale comme outil de contrôle de l'évolution de l'exploitation et du réaménagement des carrières. A notre connaissance il n'existe pas de publication faisant le point, de façon précise, sur cette question. Des recherches similaires ont été entreprises pour le contrôle de l'évolution des sites de décharges (VINCENT et al., 1985) ou de zones minières (LEGG, 1986; STOHR et al., 1987), mais les problèmes qui y ont été abordés sont assez spécifiquement liés à ces types de terrains : répercussions de l'enfouissement des déchets sur les caractéristiques du sol et de la végétation, différentes vigueurs de la végétation sur des terrains enrichis en minerais...

Pourtant la nature numérique, répétitive et synthétique des données de satellites semble particulièrement bien adaptée à un suivi régulier à l'échelle du 1/25000 au 1/50 000 des mutations de l'occupation du sol (BALLUT et al., 1980). Par ailleurs la télédétection spatiale permet généralement de distinguer les différents types de formations végétales et leur état physiologique, que les espèces soient herbacées ou arborées (GIRARD \& GIRARD, 1988). Or la végétation, en intégrant les conditions écologiques du milieu, constitue un bon révélateur de la qualité d'un réaménagement. Nous utiliserons ce critère pour suivre l'évolution des sites de carrières.

\section{MATÉRIEL ET MÉTHODES}

\section{A. Images satellites utilisées}

Pour des milieux comme les zones d'exploitation de carrières, qui sont très contrastés, hétérogènes, et oủ les différentes unités étudiées font souvent de 1 à 2 ha, il était indispensable de travailler avec des données SPOT (planche la) dont la résolution spatiale $(20 \mathrm{~m})$ est meilleure que celle de LANDSAT Thematic Mapper (TM) $(30 \mathrm{~m})$. Cependant SPOT n'ayant été lancé qu'en février 1986, nous ne disposions pas d'un recul suffisant dans le passé pour observer des transformations notables. C'est pourquoi nous avons opté pour une étude multidate (1984-1986) et multisatellite (TM et SPOT) qui porte sur la vallée de la Seine : plaine de Triel (ROTBARDT et al., 1984).

Pour comparer les 2 images pixel à pixel, nous avons effectué un calage géométrique des données TM et SPOT sur un fond topographique au 1/50 000. Puis les données TM ont été rééchantillonnées à $20 \mathrm{~m}$, en redécoupant chaque carré de 4 pixels en carrés de 9 pixels. La radiométrie affectée à ces nouveaux pixels est une moyenne pondérée des valeurs des anciens pixels, en fonction des recouvrements existants d'une grille à l'autre. Ces différents traitements nécessitent des capacités de calcul importantes et des logiciels très performánts.

\section{B. Description des sites-tests}

Pour pouvoir interpréter les images satellites, il était indispensable de disposer de zones de référence dont les caractéristiques de terrain soient parfaitement connues. Ces sites-tests étudiés sur le terrain devaient répondre aux 5 critères suivants:

- présenter une homogénéité physionomique de la végétation sur l'ensemble de la surface du site,

- posséder une végétation qui ne soit ni rarissime pour la zone étudiée, ni de transition entre 2 types de formations,

-- se situer dans un environnement permettant un repérage facile sur les images satellites,

- avoir une surface supérieure à 3 ha afin de disposer, après élimination des pixels mixtes de bordure, d'un minimum de 50 pixels $(20 \mathrm{~m} \times 20 \mathrm{~m})$ permettant de calculer les caractéristiques statistiques des valeurs radiométriques du site-test,

- ne pas avoir évolué de façon notable entre 1984 et 1986, afin de correspondre à la même formation végétale aux 2 dates. De cette façon les différences radiométriques observées sur le site correspondent essentiellement aux variations phénologiques. Ceci permet de quantifier la différence radiométrique minimale pouvant exister entre les 2 images. Toute portion d'image présentant une différence radiométrique plus importante entre les 2 dates sera passée d'une formation végétale à une autre.

L'examen de photographies aériennes IGN de 1982 nous a permis de sélectionner une centaine de zones, dont 44 seulement, répondant aux 5 critères précédents, ont été retenues après une étude de terrain. Pour ces 44 sites-tests, nous avons décrit la physionomie de la végétation en notant son organisation horizontale et verticale, ainsi que les espèces les plus couvrantes et les principales conditions écologiques du lieu.

Pour garantir la fiabilité de notre étude, ces sites-tests couvrent l'ensemble de la diversité des formations végétales que nous avons rencontrées sur le terrain. Elles se regroupent en 14 unités différentes caractérisées par le recouvrement des diverses strates, et une physionomie particulière (tabl. 1, planche 1a).

\section{Elaboration d'une méthode de suivi des réaménage- ments}

La réponse spectrale d'une formation végétale donnée, varie au cours de l'année en fonction des stades phénologiques des espèces qui la composent, sans qu'il $\mathrm{y}$ ait forcément changement du type d'occupation du sol, d'où la complexité des études multidates en télédétection (BEAudoIn et al., 1986). Pour surmonter cette difficulté, nous avons recherché les traitements permettant de différencier au mieux les véritables évolutions de l'occupation du sol. Nous avons réalisé des interprétations visuelles et automatiques en envisageant 2 approches :

- comparaison des 2 scènes,

- élaboration d'un document synthétique multidate.

\section{Interprétation visuelle}

Les différences de valeurs spectrales, enregistrées dans un canal peuvent être traduites par la gradation d'une 
TABLEAU 1

Caractéristiques de la physionomie de la végétation des différentes unités de terrain étudiées.

Vegetational characteristics of the different field units studied.

Unité de terrain

Forêt de résineux

Forêt de feuillus

Forêt dégradée

Forêt ouverte

Friche arborescente

Friche buissonneuse

Friche hétérogène

Friche avec buissons

Friche herbacée

Prairie

Pelouse

Végétation pionnière

Sol nu

Eau

\begin{tabular}{ccc}
\multicolumn{3}{c}{ Recouvrement des strates en $\%$} \\
arbore & & herbacé \\
\hline $85-100$ & $5-75$ & $5-75$ \\
$85-100$ & $5-75$ & $5-75$ \\
$25-85$ & $75-100$ & $5-75$ \\
$25-85$ & $5-75$ & $75-100$ \\
$5-25$ & $25-75$ & $25-75$ \\
$0-5$ & $50-100$ & $5-75$ \\
$0-5$ & $50-75$ & $75-100$ \\
$0-5$ & $25-50$ & $75-100$ \\
$0-5$ & $0-25$ & $85-100$ \\
$0-5$ & $0-5$ & $85-100$ \\
0 & 0 & $95-100$ \\
0 & $0-25$ & $25-50$ \\
0 & $0-25$ & $0-25$ \\
0 & 0 & 0
\end{tabular}

Particularité de la physionomie de la végétation

plus des $2 / 3$ des arbres sont des résineux plus des $2 / 3$ des arbres sont des feuillus

végétation non entretenue par l'homme

végétation entretenue par l'homme

végétation dense, rase et régulièrement entretenue couleur. En superposant 3 canaux auxquels on affecte, par exemple, les couleurs, bleu, vert et rouge on obtient diverses compositions colorées.

La comparaison visuelle de 2 compositions colorées correspondant à chaque date est fastidieuse. En effet les différences de couleur observées peuvent être dues soit à des variations phénologiques, soit à des variations dans les conditions de prise de vues, soit au procédé même de restitution papier.

C'est pourquoi nous avons étudié des compositions colorées multidates, combinant différents canaux provenant des 2 scènes (JAKOB et al., 1982 ; SCHREIER et al., 1982 ; SAINT et al., 1984 ; KING, 1985). Sur ces documents les changements radiométriques se traduisent par des couleurs particulières et peuvent être ainsi facilement repérés. Par contre l'origine de ces changements ne peut être déterminée qu'en se reportant aux images monodates ou aux observations de terrain. En effet l'interprétation des couleurs obtenues sur les images multidates est complexe car elle résulte de la combinaison des réponses spectrales des pixels à chaque date.

A partir de 6 canaux de TM et des 3 canaux SPOT on peut obtenir :

$$
C_{6}^{1} \times C_{3}^{2} \times 3 !+C_{6}^{2} \times C_{3}^{1} \times 3 !
$$

soient 378 compositions colorées diachroniques. Après de nombreux essais, nous avons sélectionné la combinaison: canal rouge (XS2) codé en bleu, canal infrarouge (XS3) codé en vert, et canal infrarouge moyen réflectif (TM5) codé en rouge (planche 1b).

Cette image présente une bonne résolution spatiale due aux canaux SPOT, et une grande richesse d'information liée aux 3 gammes de longueur d'onde. Celles-ci, faiblement corrélées, sont les plus performantes pour une étude de l'occupation du sol.

\section{Interprétation automatique}

L'interprétation automatique s'appuie sur un regroupement des pixels en un nombre restreint de classes afin d'obtenir une représentation synthétique des données radiométriques. Parmi les nombreux traitements existants, nous avons retenu une classification préassistée, bien adaptée aux études de la végétation (LACAZE, 1975).

A chacune des 14 classes définies lors de l'étude de terrain sont associés tous les sites-test connus qui s'y rapportent. Ces regroupements de sites-test, appelés « zones d'apprentissage » vont servir à classer l'ensemble des pixels de l'image. En effet par un calcul de distance des valeurs radiométriques des pixels et des zones d'apprentissage, on va pouvoir affecter chaque pixel à la classe dont il est le plus proche. La formule de calcul utilisée est :

$$
\mathrm{D}(\mathrm{k})=\left[\sum_{\mathrm{i}=1}^{\mathrm{n}}((\mathrm{x}(\mathrm{i})-m(\mathrm{k}, \mathrm{i})) / \mathrm{s}(\mathrm{k}, \mathrm{i}))^{2}\right]^{1 / 2}
$$

où $\mathrm{D}(\mathrm{k})=$ distance radiométrique du pixel à la classe $\mathrm{k}$,

$\mathrm{n}=$ nombre de canaux prix en compte,

$\mathrm{x}(\mathrm{i})=$ valeur radiométrique du pixel dans le canal $\mathrm{i}$, $\mathrm{m}(\mathrm{k}, \mathrm{i})=$ moyenne des valeurs radiométriques de la zone d'apprentissage $\mathrm{k}$ dans le canal $\mathrm{i}$, $\mathrm{s}(\mathrm{k}, \mathrm{i})=$ écart-type des valeurs radiométriques de la zone d'apprentissage $k$ dans le canal $i$.

Une première possibilité pour réaliser notre étude multidate était d'effectuer une seule classification prenant en compte les valeurs radiométriques dans les canaux provenant des 2 images. Pour être assuré de classer convenablement les différents types de changements d'occupation du sol, il fallait envisager autant de classes qu'il existe d'évolutions possibles: soit $\mathrm{n} \times \mathrm{n}$, pour des classifications monodates en $\mathrm{n}$ classes. Or au-delà de 15 à 20 classes, les confusions deviennent très importantes dans une classification. Cette méthode ne nous permettant d'envisager qu'un nombre très limité de classes d'occupation du sol a donc été écartée.

Nous avons donc procédé à 2 classifications, l'une avec les données TM, l'autre avec les données SPOT. Ces classifications ont été réalisées avec le même réfé- 

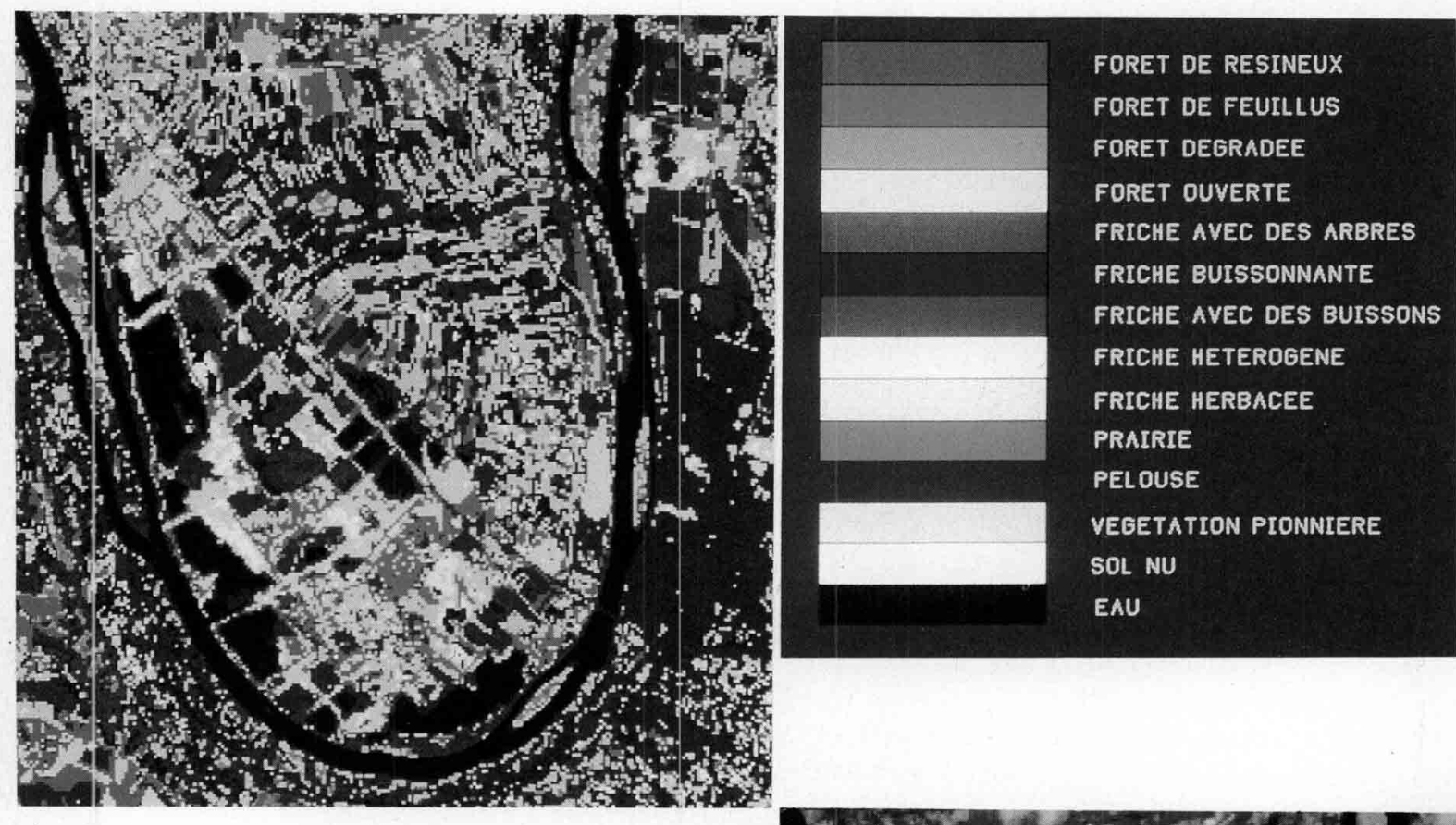

FORET DE RESINEUX

FORET DE FEUILLUS

FORET DEGRADEE

FORET OUVERTE

FRICHE AVEC DES ARBRES

FRICHE BUISSONNANTE

FRICHE AVEC DES BUISSONS

FRICHE HETEROGENE

FRICHE HERBACEE

PRAIRIE
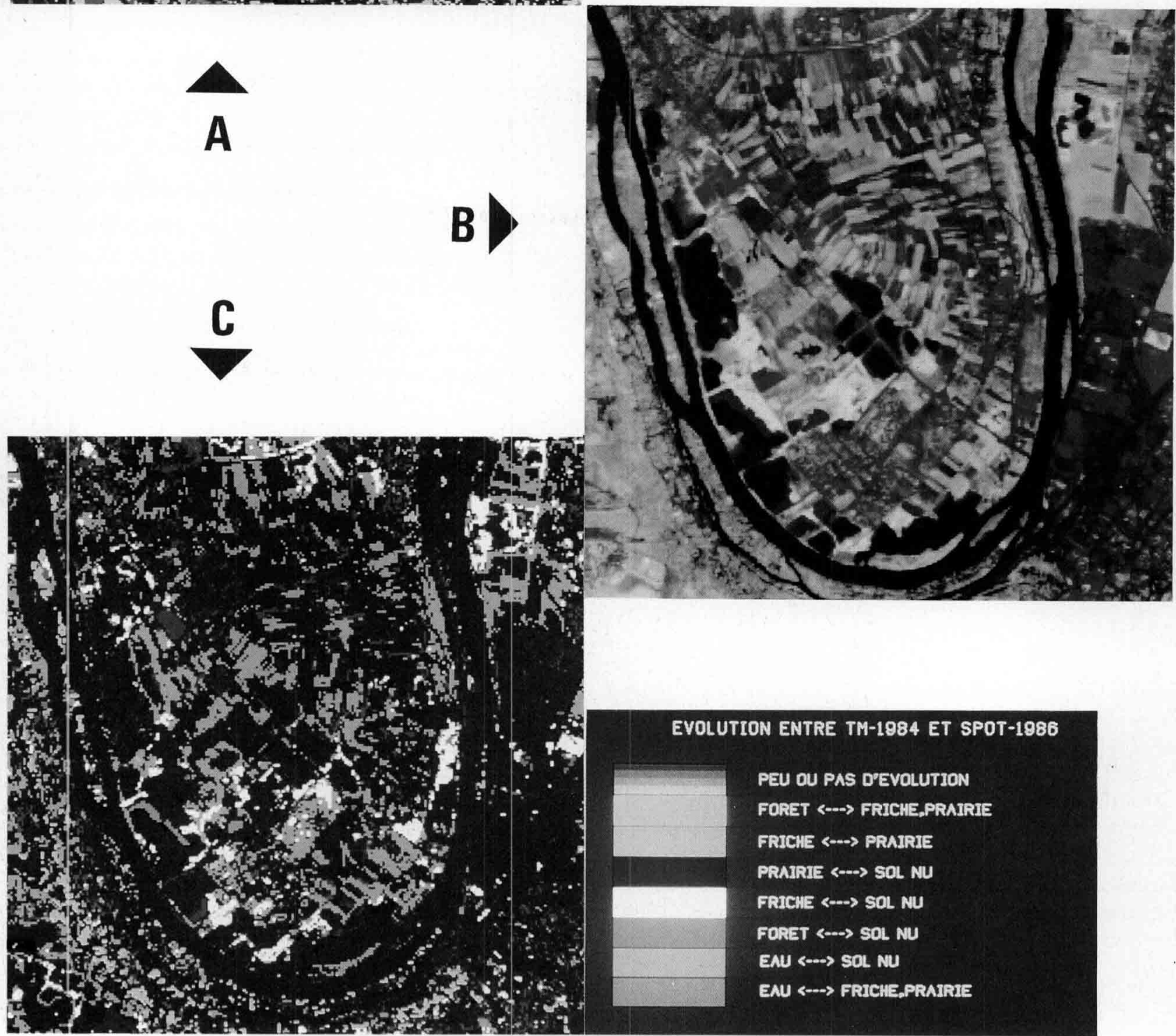
rentiel de terrain choisi pour sa stabilité entre les 2 dates d'acquisition des données satellitaires. Ainsi les 2 documents obtenus représentent l'occupation du sol respectivement en 1984 et 1986, avec des classes ayant des significations semblables dans les 2 cas.

De ce fait, l'évolution d'une zone se traduit par son passage d'une classe à l'autre, de même qu'inversement un changement de classe ne peut correspondre qu'à un changement d'occupation du sol, ou à un bouleversement important de la végétation : attaque phytosanitaire, incendie... puisqu'avec cette méthode, les changements phénologiques normaux sont intégrés au niveau des sites-tests (planche 1c).

\section{RÉSULTATS}

\section{A. Validité des classifications monodates}

Avant de discuter les classifications multidates, il est essentiel de vérifier la validité des classifications monodates, nous avons utilisé pour cela 3 traitements complémentaires entre eux.

\section{Analyse en composantes principales des valeurs radio- métriques des sites-tests}

Ayant défini les zones d'apprentissage à partir des données de terrain, il était intéressant de comparer cette classification des sites-tests avec les résultats obtenus par une analyse en composantes principales, des valeurs radiométriques. Ces analyses, présentées figure 1, mettent en évidence une bonne relation entre les comportements spectraux des sites et les caractéristiques physionomiques de la végétation.

\section{Calcul des matrices de confusion des classifications}

Après avoir vérifié la convergence entre la classification établie à partir des observations de terrain et celle basée sur les valeurs radiométriques, nous avons analysé les confusions pouvant exister entre les classes en calculant les matrices de confusion qui donnent le pourcentage de pixels affectés à chacune d'entre elles.

Ce calcul a mis en évidence un certain nombre de confusions qui pourraient être réduites en éliminant les sites-tests les plus hétérogènes et en cherchant de nouveaux mieux ciblés. Afin de garantir l'exactitude des résultats obtenus lors de la comparaison automatique des 2 classifications, nous avons réduit le nombre de classes.

5 classes ont été retenues correspondant aux grands thèmes: forêt, friche, prairie, végétation clairsemée (couvert végétal $<50$ p. 100 ), eau. En considérant les critères habituellement utilisés pour juger la qualité des matrices de confusion (QUoRum ConsultanTs, 1981) on peut estimer que la classification est fiable. En effet (tabl. 2) :

- aucune zone d'apprentissage n'a moins de 82 p. 100 des pixels bien classés (la norme inférieure couramment admise est de 60 p. 100),

- 95 p. 100 des pixels sont bien classés dans la classification faite à partir des données TM et 92 p. 100 dans le cas de SPOT (le minimum requis est généralement 90 p. 100).

Les principales confusions que nous avons constatées ont pour origine :

- similitude des réponses spectrales de l'eau et des résineux, avec de faibles réponses dans l'ensemble des canaux,

- existence d'une continuité physionomique entre forêt ouverte et friche arborescente d'une part et friche herbacée et prairie d'autre part,

- présence de pixels mixtes eau-végétation qu'il n'était pas toujours possible d'éviter lors de la délimitation des sites-tests.

\section{Sites-tests de contrôle}

En vue d'une première validation des classifications obtenues, nous avons analysé 10 sites-tests totalement indépendants des précédents en les choisissant dans une zone d'exploitation du gypse au nord-est de Paris, pour laquelle nous disposions de toutes les données de terrain requises. Nous en avons retenu 4 en forêt, 4 en friche, 2 en prairie. En tout 2 sites se sont trouvés mal classés dans la classification effectuée à partir des données TM.

Le premier correspondait à une parcelle de céréales classée en forêt de feuillus, cela s'explique par le fait que les sites-tests ont été choisis pour classer de la végétation. semi-naturelle et non des cultures.

Le second correspondait à une plantation de robiniers d'une trentaine de $m$ de large. La limite des pixels tombant juste au milieu de cette plantation, le signal enregistré par le satellite combinait les caractéristiques spectrales de la forêt et du bitume de la route qui la longe.

Planche 1

Photographies d'écran des principaux types de documents obtenus à partir de l'analyse des images Thematic Mapper du 30 avril 1984 et SPOT du 2 août 1986. Ces images couvrent l'une des boucles de la Seine à l'Ouest de Paris entre les communes d'Achères et de Triel sur Seine.

TV photographs of the main documents from TM (April 30 1984) and SPOT (August 2 1986) data analysis. These images cover one loop of the river Seine, in the western Paris region, between the towns of Achères and Triel sur Seine.

a) Classification monodate 1986 en 14 classes, réalisée à partir des données des 3 canaux SPOT.

a) 1986 monodate classification with 14 classes obtained from the 3 SPOT channels data.

b) Composition colorée multidate 1984-1986: TM5/XS3/XS2. En comparant cette composition colorée avec l'image des changements, on peut déterminer les couleurs qui correspondent à des changements.

b) Multidate color composite 1984-1986: TM5/XS3/XS2. Comparison of this color composite with the image of changes, colors corresponding to changes may be determined.

c) Image des changements obtemue par la différence des 2 classifications monodates 1984 et 1986 chacune en 5 classes.

c) Image of changes resulting from the difference of the 2 monodate classifications (1984 and 1986) thresholded in 5 classes each. 

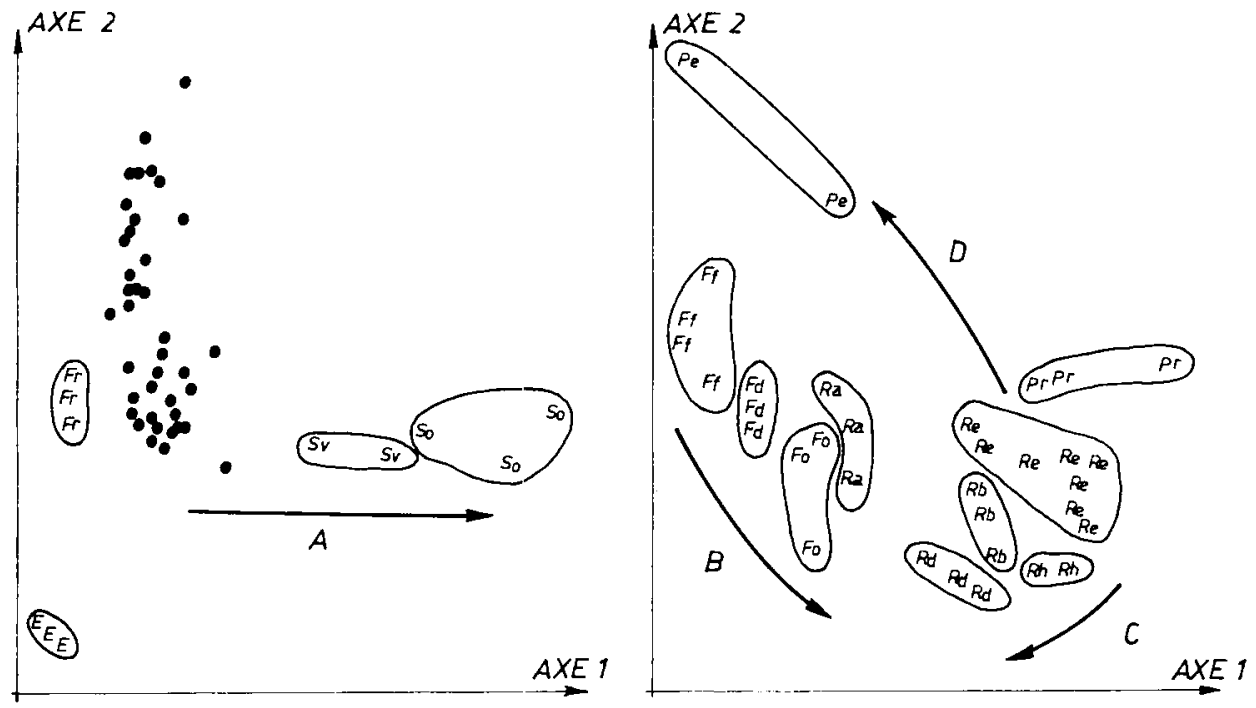

Figure 1

Analyse en composantes principales des valeurs radiométriques des sites-tests mettant en évidence la relation existant entre les comportements spectranx et les caractéristiques physionomiques de la végétation.

Principal component analysis of radiometric values for the test sites showing the existing relationship between spectral behaviour and vegetation phisionomy.

a) Plan 1-2 de l'ACP complète.

Plane $1-2$ of complete PCA

b) Plan 1-2 de l'analyse partielle.

Plane $1-2$ of limited PCA.

A diminution du recouvrement de la végétation

decrease of vegetation cover

$B$ ouverture de la strate arboréc

opening of tree cover

$C$ colonisation par les arbustes

shrub colonization

$D$ action anthropique

human influence

TABLEAU 2

Matrice de confusion des classifications monodates réalisées pour éablir l'image des changements. Les chiffres correspondent aux pourcentages de pixels des terrains affectés, après calcul, aux différentes classes.

Confusion matrix for monodate classifications performed to estahiish the image of changes. Figures correspond to the \% of field pixels assigned, after computation, to the different classes.

\section{UNITÉ DE TERRAIN}

Forêt de résincux Forêt de feuillus

Forêt dégradée Forêt ouverte

Friche arborescente

Friche buissonncuse

Friche avec buissons

Friche hétérogène

Friche herbacée

Prairie

Végétation pionnière

$$
\text { Sol nu }
$$

$\begin{array}{rrrrrr}\begin{array}{r}\text { Nombre } \\ \text { de pixels }\end{array} & & & & & \\ 188 & 86 & 0 & 0 & 0 & 13 \\ 314 & 95 & 0 & 0 & 0 & 4 \\ 195 & 92 & 8 & 0 & 0 & 0 \\ 412 & 85 & 9 & 4 & 0 & 1 \\ 276 & 9 & 91 & 0 & 0 & 0 \\ 224 & 0 & 85 & 1 & 1 & 13 \\ 311 & 0 & 95 & 5 & 0 & 0 \\ 241 & 0 & 99 & 1 & 0 & 0 \\ 303 & 0 & 88 & 6 & 0 & 6 \\ 210 & 0 & 9 & 91 & 0 & 0 \\ 330 & 1 & 7 & 91 & 1 & 0 \\ 90 & 0 & 0 & 0 & 99 & 0 \\ 203 & 0 & 0 & 0 & 99 & 1 \\ 387 & 0 & 11 & 0 & 0 & 88\end{array}$

387

88

Foret Friche Prairie Sol Eau Inclasse

$\begin{array}{rr}89 & 0 \\ 98 & 1 \\ 90 & 5 \\ 82 & 12 \\ 10 & 89 \\ 0 & 83 \\ 0 & 96 \\ 0 & 97 \\ 0 & 84 \\ 0 & 10 \\ 0 & 7 \\ 0 & 0 \\ 0 & 0 \\ 0 & 6\end{array}$

$\begin{array}{rrr}0 & 0 & 11 \\ 0 & 0 & 0 \\ 0 & 0 & 5 \\ 6 & 0 & 0 \\ 0 & 0 & 1 \\ 4 & 0 & 12 \\ 4 & 0 & 0 \\ 3 & 0 & 0 \\ 7 & 1 & 8 \\ 90 & 0 & 0 \\ 92 & 0 & 0 \\ 0 & 99 & 0 \\ 0 & 98 & 2 \\ 5 & 0 & 88\end{array}$




\section{B. Résultats des traitements multidates}

Nous avons pu vérifier que toutes les évolutions mises en évidence sur l'image des changements (planche 1c), correspondaient généralement à de véritables modifications de l'occupation du sol. Les exceptions sont soit des cas spatialement réduits à 1,2 ou 3 pixels et souvent liés à la différence de résolution spatiale entre TM et SPOT, soit des passages de friches herbacées à des prairies et inversement, très représentés en milieu agricole. L'absence de données de terrain suffisamment précises ne nous a pas permis de contrôler l'exhaustivité de notre recensement des évolutions.

Lors de notre travail de vérification des évolutions, nous avons constaté une grande complémentarité des informations apportées par la composition colorée multidate et l'image classée diachronique, appelée image des changements. Cette complémentarité est liée au fait que ces documents, issus de la même information radiométrique de départ, correspondent à 2 approches très différentes. Dans la composition colorée nous avons simplement cherché à traduire au mieux les valeurs radiométriques par des couleurs, alors que dans l'image des changements nous avons interprété ces valeurs regroupées en classes.

Une première validation de l'image classée diachronique a été réalisée en contrôlant que les changements mis en évidence sur cette image se traduisaient bien par des couleurs particulières sur la composition coloré multidate : par exemple le passage de friche à plan d'eau qui s'exprime en rouge. Par ailleurs certaines nuances qui avaient disparu lors des classifications sont mises en évidence sur la composition colorée multidate. Le passage de la friche au sol nu (en blanc sur l'image des changements) se traduit sur la composition colorée : en bleu quand il s'agit de graviers, en rose bleuté pour la terre nue, en rose pour le bitume.

Si l'image des changements est plutôt moins précise que la composition colorée, elle est par contre plus fonctionnelle en mettant en évidence les différents types de changements d'occupation des sols. De plus les surfaces couvertes par ces types peuvent être facilement évaluées par les statistiques de chacune des classes. Le report sur un fond topographique IGN au 1/50 000 des changements ainsi mis en évidence, a permis d'obtenir une cartographie de l'évolution de cette zone entre 1984 et 1986 .

\section{CONCLUSION}

La cartographie au 1/50 000 et plus encore la quantification des évolutions de l'occupation du sol que nous avons obtenues, auraient été probablement plus longues et plus coûteuses à réaliser par des méthodes conventionnelles. Dans ce domaine la télédétection spatiale devrait affirmer sa supériorité dans les années à venir. Le satellite SPOT 1 (suivi par SPOT 2 et 3 ) prend régulièrement des images qui permettront bientôt de réaliser des suivis sur des périodes plus ou moins longues tout en bénéficiant d'une résolution à $20 \mathrm{~m}$, et même à $10 \mathrm{~m}$ avec le panchromatique actuel ou le canal rouge de SPOT 3.

Cette première étude sur les possibilités de suivi par télédétection spatiale de l'évolution de la végétation en zone de carrières est forcément limitée : nous n'avons pas utilisé les possibilités du mode panchromatique, nous avons dû limiter notre typologie à 5 classes, restreindre la zone d'étude à quelques dizaines de $\mathrm{km}^{2}$. Néanmoins les résultats présentés peuvent être facilement obtenus sur d'autres sites de la région Ile-deFrance. L'élaboration d'une classification comportant plus de catégories permettrait d'envisager le suivi des ouvertures, des progrès d'exploitation et du réaménagement des carrières... La réussite de ces travaux est conditionnée par le soin apporté à la phase de terrain permettant d'acquérir le référentiel de sites-tests, représentatif des thèmes étudiés.

Enfin la méthode de suivi que nous avons présentée peut s’appliquer plus généralement aux zones de friches péri-urbaines qui présentent des caractéristiques semblables à celles des milieux que nous avons étudiés : hétérogènes, contrastés, petites tailles, changements rapides et très divers. Ces zones intermédiaires entre l'urbain et le rural posent d'importants problèmes d'aménagement. Leur suivi par télédétection serait à étudier plus en détail.

$$
\begin{aligned}
& \text { Reçu le } 2 \text { décembre } 1987 \\
& \text { Accepté le } 4 \text { arril } 1988
\end{aligned}
$$

\section{REMERCIEMENTS}

Les différents traitements mentionnés ont été réalisés a EurosenseBelfotop en Belgique dans le cadre d'un contrat entre I'Institut d'Aménagement et d'Urbanisme de la Région Ile-de-France et la Société Française d'Etudes et de Recherches Economiques et Statistiques. Que ces différents organismes soient ici vivement remerciés.

\section{RÉFÉRENCES BIBLIOGRAPHIQUES}

Ballut A., Lecup J., Lenco M., 1980. Interprétation visuelle d’une image fausse-couleur améliorée. Landsat MSS pour lc repérage des carrières et gravières in "Utilisation d'image satellite pour décrire l'état et le suivi de l'occupation du sol et de la végétation en He-de-France". IAURIF et DAV, $69 \mathrm{p}$.

Beaudoin A., Brochu R., Morin D., 1986. Détection des changements d'utilisation du sol dus à l'urbanisation, à l'aide de l'analyse en composantes principales de données multidates. Landsat - Canadian Journal of Remote Sensing, vol. $12 n^{\circ}, 29-38$.

Bournerias M., 1959. Le peuplement des espaces nus. Essais expérimentaux sur la genèse de divers groupements pionniers. Mémoire de la Société Botanique de France, Paris, 300 p.

Colmenero M., 1980. Les carrières et la réforme du code minier : aspects juridiques et techniques. Equipement mécanique. Carrières et matériaux. Edition du Moniteur. Numéro hors série, avril 80, 55 p. + annexes.
Fedoroff N., 1977. Les zones naturelles d'équilibre de la région Ilede-France. Hurepoix: Potentiels du milieu naturel. IAURIFADEPRINA, Paris, $239 \mathrm{p}$.

Girard M. C., Girard C. M., 1988. Télédétection appliquée: zones tempérées et intertropicales. Masson, éd. Paris.

Jakob B., Lamp J., Lehner M., 1982. Computer-aided soil evaluation methods on Landsat imagery in cultured landscapes. Symp. CVII. ISPRS. Toulouse, 397-405.

King C., 1985. Etude des sols et des formations superficielles par télédétection. Documents du BRGM, ${ }^{\circ} 96,174 \mathrm{p}$. + annexes.

Lacaze B., 1975. Le traitement des données multispectrales acquises par télédétection : applications à l'étude de la végétation. Thèse de Docteur Ingénieur Université des Sciences et Techniques du Languedoc: Montpellier, $140 \mathrm{p}$. + annexes.

Legg C. A., 1986. Monitoring of open-cast coal mining and reclama- 
tion works in the United Kingdom using MSS and TM imagery. Twenticth international sinposium on remote sensing of chviroment ERIM, Nairobi 86

Quorum Consultants, 1981. Les satellites au service de l'environnement. Guide pratique de télédétection spatiale, Paris, $210 \mathrm{p}$. + annexes.

Rotbardt A., Dubois J. L., Spanns M., 1984. Propositions pour la maitrise de l'évolution de la plaine de Triel-sur-Seine. Etude de l'Institut d'Aménagement et d'Urhamisme de la Région d'lle-de-France. Paris. $25 \mathrm{p}$. + annexes.

Saint G., Podaire A., Fournier Ph., Meyer-Roux J., Cordier P., 1984. Comparaison des capteurs SPOT, HRV et Landsat TM pour l'obtention de statistiques agricoles. In $I^{\prime \prime} \mathrm{Coll}$. Int. Sign. Spectrales. Colloques de I'I.N.R.A., n² 23, 149-158.

Sauveterre Ingénicur conseil, 1985. Evaluation des potentialités écolo- giques des sites de carrières après exploitation et modalités de leur restauration écologique. Document de synthèse, Ministère de l'Environnement. Taxe Parafiscale sur les Granulats, 74 p.

Schreier M., Goodfellow L. C., Lavkulich L. M., 1982. The use of digital multidate Landsat imagery in terrain classification. Photog. Engng. and R. S. 48 (1), 111-119.

Stohr C., Su W. J., du Montelle P. B., Griffin L. A., 1987. Remotesensing investigations at a hazardous-waste landfill. Photog. Engng. and R. S.. 53 (11), 1555-1563.

Vincent P., Boissonnault P., Bonn F., 1985. Analyse des sites d'enfouissement des déchets par télédétection. Télédétection et gestion des ressources: laspect opérationnel. Comptes rendus du $5^{c^{\prime}}$ congrès de I'Association Qúhécoise de Télédétection, 455-470. 